\title{
Preisverleihung
}

\section{Hilfsorganisation Mercy Ships}

Seit 15 Jahren engagiert sich die Dürr Dental AG aus Bietigheim-Bissingen für die Hilfsorganisation Mercy Ships. Dieses Engagement wurde beim 10. Lea Mittelstandspreis für soziale Verantwortung in Baden-Württemberg, am 5. Juli 2016 im

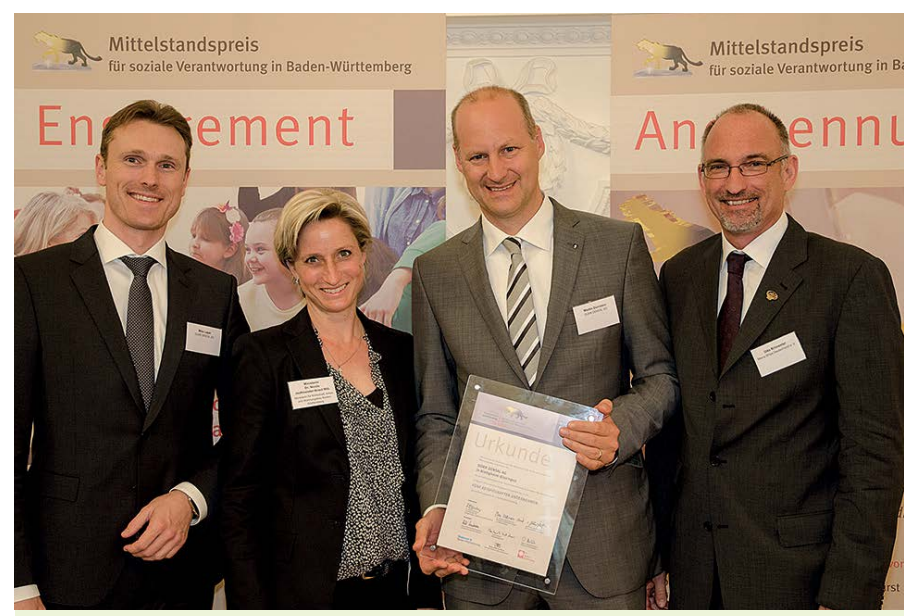

v.r. Udo Kronester, Geschäftsführer Mercy Ships Deutschland e.V., Martin Dürrstein, Vorstandsvorsitzender Dürr Dental AG, Dr. Nicole HoffmeisterKraut, Ministerin für Wirtschaft, Arbeit und Wohnungsbau in BW, Mike Layer, Leitung Zentrales Marketing Dürr Dental AG.
Neuen Schloss in Stuttgart gewürdigt. Lea steht für Leistung, Engagement und Anerkennung. Caritas und Diakonie sowie das Ministerium für Wirtschaft, Arbeit und Wohnungsbau in Baden-Württemberg zeichnen mit dem Preis das gesellschaftliche Engagement kleinerer und mittlerer Unternehmen aus.

Das Unternehmen engagiert sich für die 1978 gegründete Hilfsorganisation Mercy Ships, die in Afrika mit der Africa Mercy das größte private Hospitalschiff betreibt. Mercy Ships führt an Bord dringend indizierte fachchirurgische Eingriffe durch. Darüber hinaus werden die Gesundheitssysteme in den Gastländern durch professionelle Fortbildungsmaßnahmen gefördert. Auf dem Schiff mit 400 Personen Besatzung arbeiten pro Jahr rund 1600 Freiwillige aus insgesamt mehr als 40 Nationen.

Das Familienunternehmen stattet die Hilfsorganisation mit Produkten für seine mobilen Zahnkliniken aus. Darüber hinaus wurde mehrmals die Aktion „Ein neues Lachen für Afrika“ durchgeführt. Um die zahngesundheitliche Situation in Westafrika ein Stück weit zu verbessern, spendet das Unternehmen für verkaufte Produkte Zahnbehandlungen. Um weitere Zahnärzte und deren Teams für den Einsatz auf der Africa Mercy zu motivieren wurden Infoabende durchgeführt. Ein Benefizabend mit Geschäftspartnern führte zuletzt zu einem Spendenerlös von $70000 €$ an Mercy Ships. Neben den Tausenden von kostenlosen, oft komplizierten Operationen an Bord führt Mercy Ships in Zusammenarbeit mit einheimischen Partnern in den Dörfern der Umgebung notwendige Entwicklungsprojekte durch. Somit leistet die Organisation wichtige medizinische Hilfe, liefert aber auch Hilfsgüter und betreibt Entwicklungshilfe vor Ort, um dadurch eine langfristige und nachhaltige Veränderung in den besuchten Ländern zu ermöglichen.

Nach einer Pressemitteilung der Dürr Dental AG, Bietigheim-Bissingen www.duerrdental.com 